\title{
Determining the most significant input parameters in models of subendocardial ischaemia and their effect on ST segment epicardial potential distributions
}

\author{
Barbara M Johnston $^{\mathrm{a}, *}$, Peter R Johnston ${ }^{\mathrm{a}}$ \\ ${ }^{a}$ School of Natural Sciences and Queensland Micro- and Nanotechnology Centre, Griffith University, Nathan, Queensland, Australia, 4111
}

\begin{abstract}
There is considerable interest in simulating ischaemia in the ventricle and its effect on the electrocardiogram, because a better understanding of the connection between the two may lead to improvements in diagnosis of myocardial ischaemia. In this work we studied subendocardial ischaemia, in a simplified half-ellipsoidal bidomain model of a ventricle, and its effect on ST segment epicardial potential distributions (EPDs). We found that the EPD changed as the ischaemic depth increased, from a single minimum (min1) over the ischaemic region to a maximum (max) there, with min1 over the border of the region. Lastly, a second minimum (min2) developed on the opposite side of the ischaemic region, in addition to min1 and max. We replicated these results in a realistic ventricular model and showed that the min 1 only case could be found for ischaemic depths of up to around $35 \%$ of the ventricular wall. In addition, we systematically examined the sensitivity of EPD parameters, such as the potentials and positions of min1, max and min2, to various inputs to the half-ellipsoidal model, such as fibre rotation angle, ischaemic depth and conductivities. We found that the EPD parameters were not sensitive to the blood or transverse bidomain conductivities and were most sensitive to either ischaemic depth and/or fibre rotation angle. This allowed us to conclude that the asynchronous development of the two minima might provide a way of distinguishing between low and high thickness subendocardial ischaemia, and that this method may well be valid despite variability in the population.
\end{abstract}

Keywords: ischaemia, ST depression, ST elevation, bidomain model, Gaussian Process emulators

\section{Introduction}

Myocardial ischaemia results from a critical reduction of blood supply to the heart and/or an increase in the metabolic demand, which cannot be met by the heart [1]. When the ischaemia is transmural, clinicians are able to determine the location of the ischaemia using the elevation of the ST segment in superficial electrocardiogram (ECG) leads [2]. However this is not the case for nontransmural ischaemia.

Many attempts have been made to connect ST depression of the ECG to subendocardial ischaemia, via both experimental and modelling studies, but, to date, the results are inconclusive [3]. This is perhaps not surprising since the connection between ischaemia and ST depression in epicardial electrograms is not yet clear [2], let alone the way in which epicardial depression may translate to the body surface and hence to the ECG.

Part of the difficulty is that studies produce contradictory findings about the location of areas of epicardial ST depression and ST elevation. For example, using an experimental model, Guyton et al. [4] have shown that nontransmural ischaemia is a necessary condition for ST depression in leads overlying the ischaemic region. How-

* Corresponding author ever, it is not a sufficient condition as shown by the experimental study of $\mathrm{Li}$ et al. [5] that found ST depression, which strengthened as the ischaemia became transmural, was positioned over the lateral boundary between healthy and ischaemic tissue. The study found that this occurred regardless of whether there was ST elevation, and that ST elevation over the ischaemic region developed as the degree of ischaemia increased.

Modelling studies of ischaemia generally use the bidomain model [6] to represent the cardiac ventricular tissue, which is made up of sheets of cardiac fibres that rotate relative to one another through the wall of the ventricle. The bidomain model considers the tissue as consisting of two interpenetrating domains, intracellular $(i)$ and extracellular $(e)$, within which the electrical properties are homogenised. The electrical current is able to flow more easily along than across the fibres within a sheet, or between the sheets. These directions are designated longitudinal $(l)$, transverse $(t)$ and normal $(n)$, respectively, resulting in six bidomain conductivities $\left(g_{p q}, q=l, t, n\right)$.

A modelling study in a canine heart [7] has identified two ST segment epicardial patterns: the first corresponds to a very low $(<20 \%)$ ischaemic depth and consists of a single depression over the ischaemic region; the second is for deeper ischaemia than this and has an elevation over the ischaemic region flanked by two depressions. Later 
studies in human hearts $[8,3]$ found the single depression case could occur for very large, thin ischaemic regions and suggested that intracavitary blood may also influence the pattern.

A number of modelling studies $[9,7,8,3,10,11]$ have suggested that the anisotropy of the conductivity values is crucial to the form of the EPD, while others $[12,13]$ have shown the effect of using only four bidomain conductivity values (i.e. equating the normal and transverse conductivities) instead of the full set of six. Another suggestion that the conductivities in the ischaemic tissue may be sufficiently different from those in healthy tissue as to have an effect on the EPDs has also been investigated $[1,3]$.

More recently, an experimental study [2] has challenged the assumption that ischaemia begins in the subendocardium and has found that it arises throughout the ventricular wall.

There is considerable uncertainty in the input parameters to modelling studies such as those discussed above. This is particularly true for the bidomain conductivities, since no normal conductivity values and no consistent longitudinal and transverse conductivities have been determined experimentally $[14,15]$. In addition, conductivity values vary during the time course of ischaemia [16] and there are possibly differences between the conductivity values and fibre rotation angles between individuals.

Although some of the above modelling studies have considered the effect of varying input parameters such as blood conductivity, fibre rotation angle and bidomain conductivities, on the form of the EPD when modelling ischaemia in the ventricle during the ST segment, only one [17] has done so systematically (and included all six bidomain conductivities). Instead of performing many thousands of Monte Carlo simulations, this was achieved by constructing fast-running Gaussian Process emulators (surrogate models) [18] for the various outputs of interest (i.e. the maxima and minima in the EPDs). From this, the study was able to identify the model inputs that had the most effect on the outputs.

However, that work used a simplified model of the ventricle. The present study improves on that work by using a half-ellipsoidal model, which is a much better approximation to the left ventricle, both in size and structurally. In this study, we compare simulations, using 'mean' input values [17], in the half-ellipsoidal model with simulations in a realistic ventricular model [19], based on a canine heart. After this validation, the half-ellipsoidal model is used to determine the inputs to which the EPDs are most sensitive and examine the effect that these inputs have on the form of the EPD, in an attempt to increase our understanding of the connection between ischaemia and epicardial ST segment depression and elevation.

\section{Methods}

\subsection{Bidomain model}

We model the electric potential in cardiac ventricular tissue, during the ST segment of the ECG, using the passive bidomain equation $[6,20]$

$$
\nabla \cdot\left(\mathbf{M}_{i}+\mathbf{M}_{e}\right) \nabla \phi_{e}=-\nabla \cdot \mathbf{M}_{i} \nabla \phi_{m}
$$

where $\phi_{e}, \phi_{i}$ and $\phi_{m}=\phi_{e}-\phi_{i}$ are the extracellular $(e)$, intracellular $(i)$ and transmembrane potentials, respectively, and $\mathbf{M}_{e}$ and $\mathbf{M}_{i}$ are conductivity tensors of the form $\mathbf{M}_{p}=$ $\mathbf{A} \mathbf{G}_{p} \mathbf{A}^{T}(p=i, e)$. Here $\mathbf{G}_{p}(p=i, e)$ is a diagonal matrix that contains the bidomain conductivities $\left(g_{p q}, q=l, t, n\right)$ and $\mathbf{A}$ is a rotation matrix that maps the local fibre direction into the global coordinate system [20]. As will be discussed below, we will assume that $\phi_{m}$ is a known function of position.

\subsection{Ischaemia model}

This work will consider the acute phase of ischaemia, and the ischaemic region will be represented by a 'rectangular' slab of tissue that occupies part of the ventricular wall starting at the endocardium. This was chosen bearing in mind that previous work [19], comparing rectangular and cylindrical ischaemic regions, has shown that there is little difference in the EPDs in the two cases.

During the ST segment, we represent $\phi_{m}$ by [6]

$$
\phi_{m}(r, \theta, \phi)=\Delta \phi_{p} \Psi\left(r_{a}-r\right) \Psi\left(\theta-\theta_{0}\right) \Psi(\phi)
$$

where $\Delta \phi_{p}$ is the difference between the plateau potentials in normal and ischaemic tissue, which we set to $-30 \mathrm{mV}$ $[20,9]$. Here the ischaemic region is centred at $\left(r_{a}, \theta_{0}, 0\right)$ on the endocardial surface and

$$
\Psi(t)= \begin{cases}\frac{1-\exp \left(-a_{t} / \lambda_{t}\right) \cosh \left(t / \lambda_{t}\right)}{1-\exp \left(-a_{t} / \lambda_{t}\right)} & |t| \leq a_{t} \\ \frac{\exp \left(-|t| / \lambda_{t}\right) \sinh \left(a_{t} / \lambda_{t}\right)}{1-\exp \left(-a_{t} / \lambda_{t}\right)} & |t|>a_{t}\end{cases}
$$

where $a_{t}(t=r, \theta, \phi)$ is the half-width of the ischaemic region.

We initally set $\lambda_{t}=0.01 \forall t$ to produce a narrow border zone between the normal and ischaemic tissue [20]. We also consider the effect of using a wider border zone and also a 'new' representation chosen to more closely match experimental results [21, 22].

The 'new' version of $\Psi(t)$ is given by

$$
\Psi_{2}(t)= \begin{cases}\frac{1-2\left(1-a_{2}\right) \exp \left(-a_{1} / b_{1}\right) \cosh \left(t / b_{1}\right)}{1-2\left(1-a_{2}\right) \exp \left(-a_{1} / b_{1}\right)} & |t| \leq a_{1} \\ \frac{2 a_{2} \exp \left(-|t| / b_{2}\right) \sinh \left(a_{1} / b_{2}\right)}{1-2\left(1-a_{2}\right) \exp \left(-a_{1} / b_{2}\right)} & |t|>a_{1}\end{cases}
$$

where $a_{1}=0.9, b_{1}=0.2, b_{2}=0.01, a_{2}=0.1$ and $\phi_{m}$ is as in equation (2), but with $\Psi$ replaced by $\Psi_{2}$ for $t=\theta$ and $\phi$.

A comparison of $\phi_{m}$ for 'narrow' $\left(\lambda_{t}=0.01, t=r, \theta, \phi\right)$ and 'wide' $\left(\lambda_{r}=0.01, \lambda_{t}=0.1, t=\theta, \phi\right)$ border zones, as well as for the 'new' representation is given in Figure 1. 


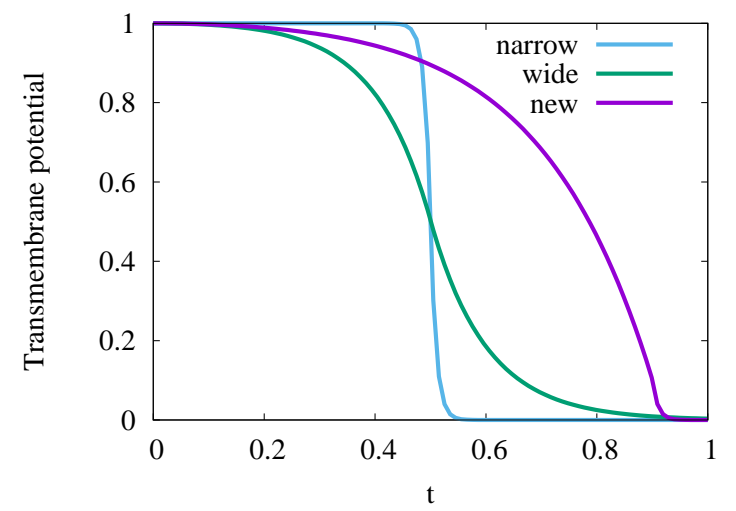

Figure 1: Transmembrane potential for the 'narrow' and 'wide' border zones, as well as for the 'new' representation (equation (4)).

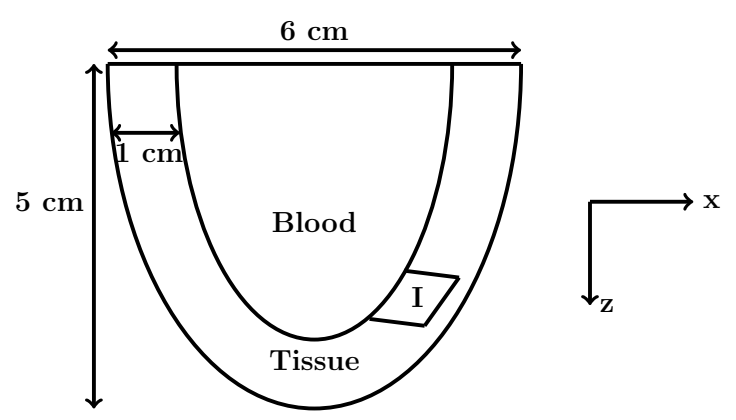

Figure 2: Cross-sectional view $(x-z$ plane at $y=0)$ of the halfellipsoidal model. Ischaemic region is marked I.

\subsection{Geometrical models}

In this work we consider two models, a half-ellipsoidal model of the left ventricle and a realistic ventricular model.

\subsubsection{Half-ellipsoidal ventricular model}

In this model we represent the left ventricle of the heart by a half-ellipsoid (Figure 2) given by the equations

$$
x=a \cos \theta \cos \phi, \quad y=a \cos \theta \sin \phi, \quad z=c \sin \theta
$$

where $0 \leq \theta \leq \pi / 2$ and $-\pi \leq \phi \leq \pi$.

We take $a=2 \mathrm{~cm}, c=4 \mathrm{~cm}$ for the endocardial surface and $a=3 \mathrm{~cm}, c=5 \mathrm{~cm}$ for the epicardial surface to give a realistically sized left ventricle, with a tissue thickness of $1 \mathrm{~cm}$ throughout. We assume that the endocardial surface is in contact with a volume of blood (Figure 2). The ischaemic region (I) is represented by a 'rectangular' patch of tissue, of ischaemic depth ISC (as a \%), given by $\left(50^{\circ} \leq \theta \leq 70^{\circ},-15^{\circ} \leq \phi \leq 15^{\circ}, 10 \% \leq \mathrm{ISC}<100 \%\right)$.

We also assume that the sheets of cardiac fibres remain parallel to the epicardium as they rotate linearly through a fibre rotation angle, represented by $\operatorname{ROT}$ in $^{\circ}$ ), between the epicardium and the endocardium [23] and we offset the cardiac fibres by $-45^{\circ}$ at the epicardium [20].

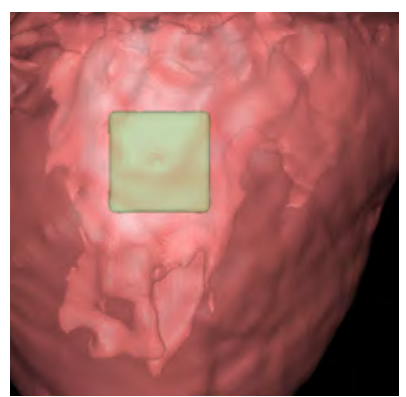

Figure 3: The realistic heart and the ischaemic region. The base to apex distance is approximately $7.6 \mathrm{~cm}$ and the average diameter near the base is approximately $8 \mathrm{~cm}$.

\subsubsection{Realistic ventricular model}

The realistic ventricular model, which will be referred to as the 'realistic model' hereafter, is based on canine geometry that is obtained from MRI data, along with fibre orientations that come from diffusion weighted images [24]. Both ventricles are filled with blood [19] and the ischaemic region in this case is 'square' in shape and extends partway through the wall of the left ventricle, beginning at the endocardium (Figure 3). This \% depth is again denoted by ISC, although in this case the value for ISC is approximate, rather than exact, because the thickness of the realistic model tissue is not quite uniform in the region where the ischaemia is located [19].

\subsection{Solution technique}

Since both models have blood-filled ventricles, we model the potential in the blood $\phi_{b}$ using Laplace's equation $\nabla^{2} \phi_{b}=0$. Both the half-ellipsoidal ventricular model and the realistic full heart model are assumed to be in contact with the air. Each model is solved using the same boundary conditions as previously [20]; that is, we assume, at the air-tissue interfaces and the blood-air interfaces, as well as at the boundary of the extra- and intracellular domains, that there is no current flux. We also assume that potential and current are continuous in the extracellular space at the blood-tissue boundary.

The half-ellipsoidal model is solved with previously validated technique $[10,20]$ that is based on the finite volume method, using a mesh that consists of 423,975 nodes and 414,832 hexahedral elements [20]. In the case of the realistic model, the solution technique is the finite volume method $[20,19]$ and the mesh contains 717,709 nodes joined by 4,486,917 tetrahedral elements.

The parameters used in the simulations are discussed in the next section.

\subsection{Analysis methods}

As mentioned in the Introduction, no definitive values are known for the bidomain conductivities $\left(g_{p q}, p=\right.$ $i, e, q=l, t, n)$. Ranges for these values, based on the literature, are discussed in [17] and are given in Table 1, along with ranges for ROT, ISC and the blood conductivity $g_{b}$. 
Table 1: Data ranges for parameters in this study. Units are degrees for fibre rotation (ROT), \% depth for ISC and $\mathrm{mS} / \mathrm{cm}$ for conductivities.

\begin{tabular}{c|ccc}
\hline Parameter & Minimum & Mean & Maximum \\
\hline ROT & 60 & 100 & 140 \\
ISC & 10 & 50 & 90 \\
$g_{b}$ & 3.25 & 6.5 & 9.75 \\
$g_{i l}$ & 1.2 & 2.4 & 3.6 \\
$g_{e l}$ & 1.2 & 2.4 & 3.6 \\
$g_{i t}$ & 0.12 & 0.24 & 0.36 \\
$g_{\text {et }}$ & 0.8 & 1.6 & 2.4 \\
$g_{\text {in }}$ & 0.05 & 0.1 & 0.15 \\
$g_{\text {en }}$ & 0.5 & 1.0 & 1.5
\end{tabular}

We create sets of design data by allowing each input in Table 1 to vary uniformly across its range (mean $\pm 0.5 \times$ mean) using a Latin Hypercube (LHC) sampling routine, which is part of the software described in the next section. These sets of input parameters are used to solve the model and to produce an EPD (see, for example, Figure $4(\mathrm{i})$ ). In this polar plot, the surface of the ellipsoid has been flattened into a circle, with the apex of the ventricle positioned at the origin. The radius of each node on the circle is scaled according to its $\theta$ value in Equation (5) and $\phi_{e}$ is set to zero at the apex of the ventricle. The features of this plot (minima and maximum) are the outputs of interest and they will be categorised by their potentials and their positions.

Two different methods, Gaussian Process emulators and Partial Least Squares regression are used to study the effect of uncertainty in the input parameters on the various outputs that characterise the EPD.

\subsubsection{Gaussian Process emulator}

We construct a Gaussian Process (GP) emulator [18], a fast surrogate of the model, using the software GP_emu_UQSA (DOI 10.5281/zenodo.215521). This package can be used to perform both uncertainty quantification (UQ) and sensitivity analysis (SA).

After generating $j=1, \ldots, N$ sets of design data $\left\{\operatorname{ROT}^{(j)}, \operatorname{ISC}^{(j)}, g_{b}^{(j)}, g_{i l}^{(j)}, g_{e l}^{(j)}, g_{i t}^{(j)}, g_{e t}^{(j)}, g_{i n}^{(j)}, g_{e n}^{(j)}\right\}$, as described in Section 2.5, the model is solved for each of these sets and $N$ EPDs are produced, from which $N$ sets of outputs are calculated. Then, for each output, an emulator is fitted to a subset of the design data (approximately $10 \%$ is kept for verification), using a Gaussian covariance function and a linear mean for the training. The accuracy of the fit of the emulator is checked against the verification dataset [18] and a final emulator is built from the combined verification plus training datasets.

The GP_emu_UQSA software is then used to produce main effect plots. These show the inputs $\mathbf{x}$ against the mean effect for a particular output $y=f(\mathbf{x})$. The mean effect is defined to be the conditional expectation of that output, conditional on the input variable $x_{w}$, after averaging over the remaining variables; that is, mean effect $=$ $E\left\{f(x) \mid x_{w}\right\}$. For a particular emulator, this is calculated for each input, by allowing $x_{w}$ to vary over [0,1], while the other inputs are taken to be independently normally distributed with a mean of 0.5 and a variance of 0.04 [18].

In addition to mean effects, we also calculated main effect sensitivity indices, which quantify the contribution of each input to each output [25]. This unsigned index is calculated as the ratio of the variance (Var) of the mean effect to the variance of the model output; that is,

$$
\text { Sensitivity }=\frac{\operatorname{Var}\left[E\left\{f(x) \mid x_{w}\right\}\right]}{\operatorname{Var}\{f(x)\}} .
$$

For more details see Chang et al. [18].

\subsubsection{Partial Least Squares regression}

Partial Least Squares (PLS) regression [26, 27] is somewhat similar to principal component analysis. Its aim is to find a set of components that simultaneously decompose both the input and output vectors, whilst ensuring that the components maximise the covariance between the inputs and outputs [26]. This approach uses the same design data as in Section 2.5.1 and again produces an emulator from which the relative effects of various inputs are calculated using the NIPALS algorithm [28, 26], as described in the study by Sobie [27]. In PLS the regression coefficients are signed and they indicate the change that would occur in an output due to a change in an input.

\section{Results}

\subsection{Effect of inputs on mean EPDs in the half-ellipsoidal model}

In Sections 3.1.1-3.1.4, we examine the effect of varying particular inputs on 'mean' EPDs, that is, those EPDs produced using the mean values from Table 1 for the remainder of the inputs in the half-ellipsoidal model. We then repeat some of this work in the realistic model. In later sections, the combined effect of varying all the inputs will be quantified via GP emulators and PLS regression for the half-ellipsoidal model.

\subsubsection{Variation of mean EPDs with ischaemic depth in the half-ellipsoidal model}

The polar plots in Figure 4, which are generated using the mean values from Table 1, except for ISC, change in character as ISC increases. In the plots the ischaemic region is outlined in white and, when it is very thin (ISC $=10 \%$, Figure 4(a)), the main feature is a minimum over the ischaemic region. As ISC increases, this minimum (min1) increases slowly in magnitude (see Table 2, which lists the potentials for this minimum (min1V) for different ischaemic depths). The position of min1 also changes as 


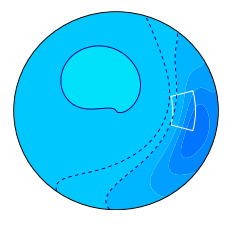

(a) $10 \%$ ischaemia

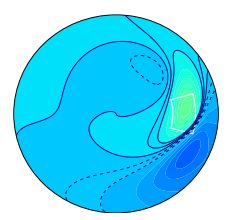

(d) $40 \%$ ischaemia

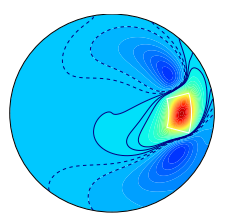

(g) $70 \%$ ischaemia

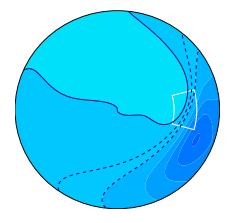

(b) $20 \%$ ischaemia

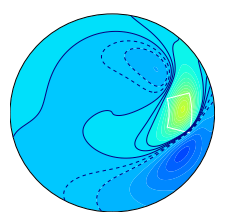

(e) $50 \%$ ischaemia

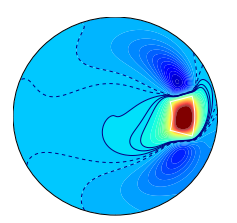

(h) $80 \%$ ischaemia

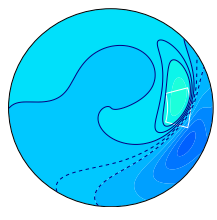

(c) $30 \%$ ischaemia

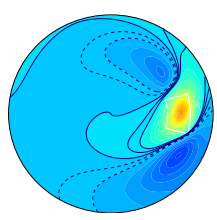

(f) $60 \%$ ischaemia

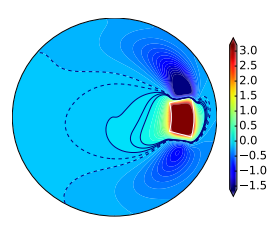

(i) $90 \%$ ischaemia
Figure 4: EPDs generated from mean values (Table 1) for a range of ischaemic depths. Dashed lines are negative potentials, solid lines positive potentials. Contours range from -0.2 to 0.1 in steps of 0.05 $\mathrm{mV}$. The ischaemic region is outlined in white.

ISC increases - it moves from over the ischaemic region clockwise to along its lateral border.

For ISC $=20-30 \%$ (Figure 4(b),(c)), a maximum (max) starts to form directly over the ischaemic region and its magnitude $(\operatorname{maxV})$ increases substantially with ISC (Table 2 ). In addition, for ISC $=30-40 \%$ and above (Figures $4(\mathrm{c})$ $4(\mathrm{j})$ ), a second minimum (min2) develops on the opposite side of the ischaemic region from min1. In this case, its magnitude (see min2V in Table 2) increases more quickly than $\min 1 \mathrm{~V}$, overtaking it from about $\mathrm{ISC}=70 \%$ onward. Also, min2 appears to rotate in a clockwise direction with increasing ISC.

In summary, increasing ISC changes the mean EPDs both quantitatively and qualitatively. Quantitatively, the magnitude of each of the features increases and their positions move clockwise with increasing ISC. Qualitatively, we can identify three 'types' of EPDs: Type 1: min1 over the ischaemic region (e.g. Figure 4(a)); Type 2: max over the ischaemic region, with min1 flanking it (e.g. Figure 4(b)); Type 3: max over the ischaemic region, with min1 and min2 flanking it on opposide sides (e.g. Figure $4(\mathrm{c})-(\mathrm{i}))$.

\subsubsection{Variation of mean EPDs with ischaemic depth in} the half-ellipsoidal model with changed parameters

Next we considered the effect of:

1. increasing the size of the ischaemic region to $\left(-20^{\circ} \leq\right.$ $\theta \leq 20^{\circ}, 50^{\circ} \leq \phi \leq 80^{\circ}$ ), which is now approximately twice as large as the original region;

2. changing the position of the ischaemic region, to (a) $10^{\circ} \leq \theta \leq 30^{\circ}$, (b) $20^{\circ} \leq \theta \leq 40^{\circ}, \ldots$ or, (f) $70^{\circ} \leq$ $\theta \leq \overline{90}$
Table 2: Potentials (in $\mathrm{mV}$ ) for various features of the EPDs in Figure 4, generated using mean values (Table 1), except for ISC, for the half-ellipsoidal model.

\begin{tabular}{c|ccc}
\hline $\begin{array}{c}\text { ISC } \\
\%\end{array}$ & $\min 1 \mathrm{~V}$ & $\operatorname{maxV}$ & $\min 2 \mathrm{~V}$ \\
\hline 10 & -0.49 & - & - \\
20 & -0.51 & 0.05 & - \\
30 & -0.54 & 0.30 & -0.02 \\
40 & -0.59 & 0.68 & -0.08 \\
50 & -0.64 & 1.22 & -0.20 \\
60 & -0.71 & 2.00 & -0.42 \\
70 & -0.79 & 2.92 & -0.77 \\
80 & -0.89 & 4.18 & -1.31 \\
90 & -1.01 & 5.73 & -2.27
\end{tabular}

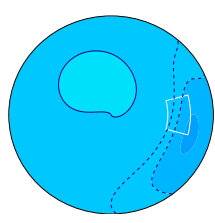

(a) $10 \%$ ischaemia

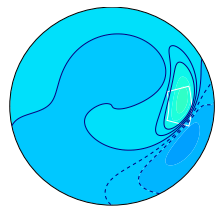

(d) $40 \%$ ischaemia

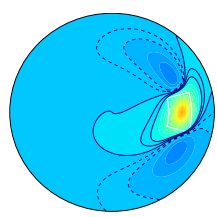

(g) $70 \%$ ischaemi

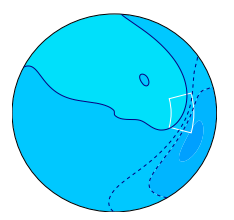

(b) $20 \%$ ischaemia

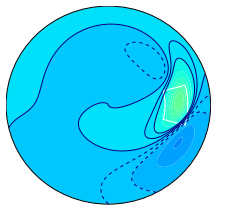

(e) $50 \%$ ischaemia

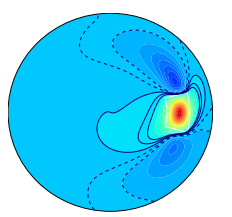

(h) $80 \%$ ischaemia

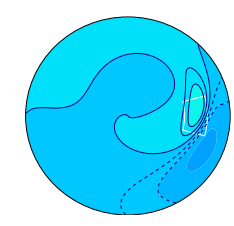

(c) $30 \%$ ischaemia

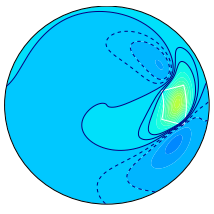

(f) $60 \%$ ischaemia

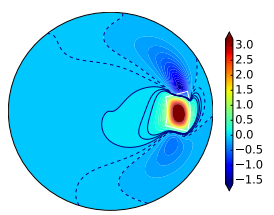

(i) $90 \%$ ischaemia
Figure 5: EPDs generated from mean values (Table 1), using the 'new' transmembrane potential given in equation (4), for a range of ischaemic depths. See Figure 4 for contour details.

3. increasing the width of the border zone between the ischaemic and healthy tissue to $\left(\lambda_{r}=0.01, \lambda_{t}=\right.$ $0.1, t=\theta, \phi) ;$ and

4. changing the representation of the transmembrane potential $\phi_{m}$ (see equation (4)).

Note, for each representation of $\phi_{m}$, we keep a narrow $\left(\lambda_{r}=0.01\right)$ border zone in the transmural direction, as to do otherwise would lead to the ischaemic region having a greater depth than was intended.

For each of scenarios (1), (2)(b)-(e), (3) and (4), the set of EPDs produced, using the mean values from Table 1 for $\mathrm{ISC}=10 \%$ to $90 \%$, is qualitatively the same as Figure 4; that is, $\min 1, \max$ and $\min 2$ are in similar positions and appear at similar values of ISC. (Cases (2)(a) 
Table 3: Potentials (in $\mathrm{mV}$ ) for various features of the EPDs in Figure 4, generated using mean values (Table 1), except for ISC, and the 'new' transmembrane potential given in equation (4), for the half-ellipsoidal model.

\begin{tabular}{c|ccc}
\hline $\begin{array}{c}\text { ISC } \\
\%\end{array}$ & $\min 1 \mathrm{~V}$ & $\operatorname{maxV}$ & $\min 2 \mathrm{~V}$ \\
\hline 10 & -0.23 & - & - \\
20 & -0.24 & 0.03 & - \\
30 & -0.26 & 0.17 & -0.01 \\
40 & -0.28 & 0.38 & -0.04 \\
50 & -0.31 & 0.70 & -0.10 \\
60 & -0.34 & 1.16 & -0.21 \\
70 & -0.38 & 1.84 & -0.40 \\
80 & -0.42 & 2.89 & -0.74 \\
90 & -0.48 & 4.57 & -1.46
\end{tabular}

Table 4: Potentials (in $\mathrm{mV}$ ) for various features of the EPDs in Figure 6, generated using mean values for conductivities (Table 1) and varying ROT and ISC in the half-ellipsoidal model.

\begin{tabular}{ccccc}
\hline ROT & ISC & $\min 1 \mathrm{~V}$ & $\operatorname{maxV}$ & $\min 2 \mathrm{~V}$ \\
\hline 60 & 10 & -0.23 & - & - \\
& 20 & -0.22 & 0.01 & -0.13 \\
& 50 & -0.35 & 1.10 & -0.53 \\
& 70 & -0.52 & 2.67 & -1.19 \\
\hline \multirow{2}{*}{100} & 10 & -0.49 & - & - \\
& 20 & -0.51 & 0.55 & - \\
& 50 & -0.64 & 1.22 & -0.20 \\
& 70 & -0.79 & 2.92 & -0.77 \\
\hline 140 & 10 & -0.68 & - & - \\
& 20 & -0.76 & 0.03 & - \\
& 50 & -0.91 & 1.18 & -0.03 \\
& 70 & -0.95 & 3.03 & -0.43
\end{tabular}

and (2)(f) are those where the ischaemic region is close to the apex or the base and, in these cases, there are sometimes greater differences in the potentials from the original $50^{\circ} \leq \theta \leq 70^{\circ}$ case). In cases (2)(b)-(e) and (3), the values of the potentials are quite similar to those in Table 3 and in case (1) the values are larger. In case (4) they are smaller (see Figure 5 and Table 3). Despite these differences, later work (Section 3.3) will show that the sensitivity results are not affected by changes in the size and location of the ischaemic region, the width of the ischaemic border, nor the change that we made in the representation of the transmembrane potential. For this reason, unless otherwise stated, the 'narrow' border zone will be used in the remainder of this work.

\subsubsection{Variation of mean EPDs with ischaemic depth and fibre rotation in the half-ellipsoidal model}

We now examine a set of EPDs where ISC and ROT are both allowed to vary, but the remainder of the inputs

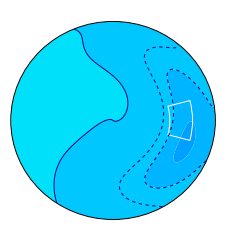

(a)

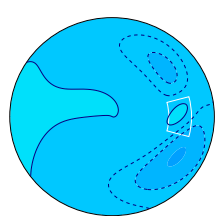

(d)

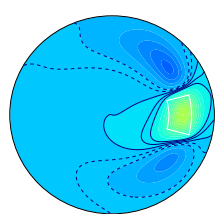

(g)

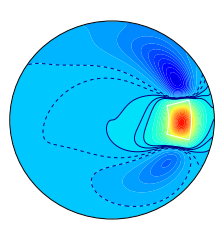

(j)

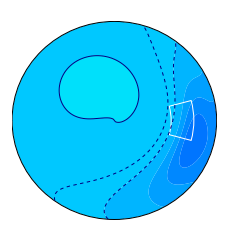

(b)

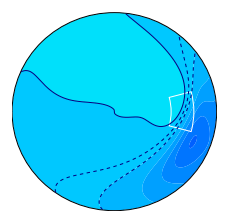

(e)

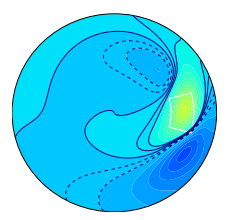

(h)

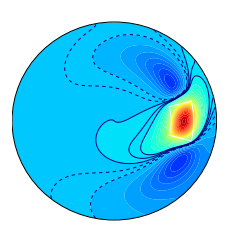

(k)

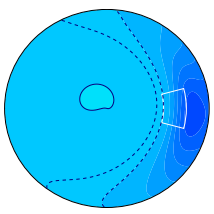

(c)

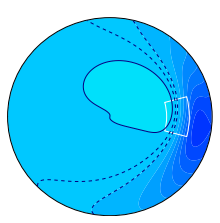

(f)

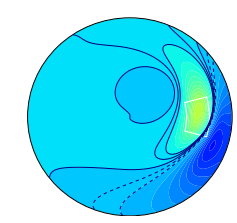

(i)

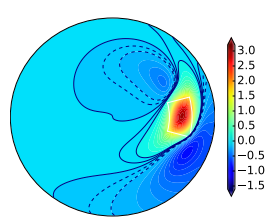

(1)
Figure 6: EPDs generated from mean values (Table 1), for ischaemic depths of $10 \%, 20 \%, 50 \%$ and $70 \%$, corresponding to the rows from top to bottom, and for fibre rotation angles of $60^{\circ}, 100^{\circ}$ and $140^{\circ}$, corresponding to the columns from left to right. See the caption of Figure 4 for contour details.

from Table 1 are set to their means. The rows in Figure 6 correspond to $\mathrm{ISC}=10 \%, 20 \%, 50 \%$ and $70 \%$, while the columns correspond to $\mathrm{ROT}=60^{\circ}, 100^{\circ}$ and $140^{\circ}$.

Values for the potentials for the various features are given in Table 4. As in Section 3.1.1, we see that increasing ISC leads to increases in the magnitudes of min1V, $\max \mathrm{V}$ and $\min 2 \mathrm{~V}$ and this holds across the range of ROT values. However, the ISC value at which min2 appears does depend on ROT and is higher for higher ROT.

In contrast, the effect of ROT for fixed ISC is different depending on the feature under consideration. For example, for all ISC values, increasing ROT leads to an increase in potential magnitude for min1, and most max cases, but has the opposite effect for min2. It would also appear that ROT affects the position of the minima. For example, if we consider $\mathrm{ISC}=70 \%$, min 1 moves anti-clockwise as ROT increases (Figure 6(j)-(l)).

In summary, both ROT and ISC affect the magnitudes of the features and ROT also appears to affect the position of $\min 1$. 


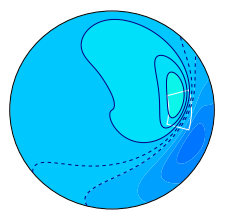

(a)

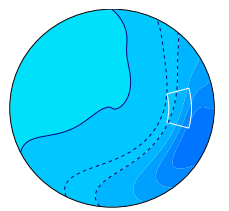

(d)

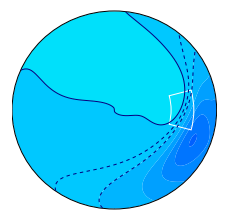

(b)

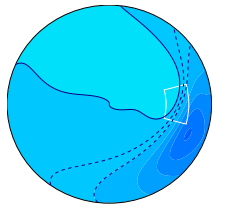

(e)

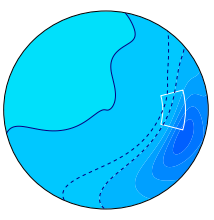

(c)

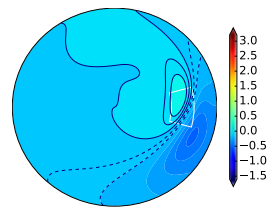

(f)
Figure 7: EPDs generated with $\mathrm{ISC}=20 \%$ and mean values for other inputs (Table 1), except for the conductivities $g_{i n}$ (top row) and $g_{\text {en }}$ (bottom row). The columns from left to right correspond to their min, mean and max values (Table 1). See the caption of Figure 4 for contour details.

\subsubsection{Variation of mean EPDs with conductivity in the half-ellipsoidal model}

The final set of 'mean' type plots that we will examine for the half-ellipsoidal model are those for $\mathrm{ISC}=20 \%$ (Figure 7), where we set all other inputs to their means (Table 1), except for a particular conductivity value. We then consider the minimum, mean and maximum for that conductivity and these correspond to the columns (from left to right) in Figure 7. The conductivities $g_{\text {in }}$ (top row) and $g_{e n}$ (bottom row) were chosen because varying their values has a qualitative effect on the form of the EPD.

For example, in Fig 7 (row 1), as $g_{\text {in }}$ is increased, the EPD changes from type 2 in (a) and (b) to type 1 in (c), whereas the opposite is true for $g_{e n}$, which is of type 1 in (d) but type 2 in (f).

\subsection{Effect of inputs on mean EPDs for the realistic model}

We now examine the effect of changes in ischaemic depth and conductivity values on the EPDs using the realistic model.

\subsubsection{Variation of mean EPDs with ischaemic depth for the realistic model}

We repeated the study of Section 3.1.1, which examined the effect on mean EPDs as ISC varied from 10-90\%, this time for the realistic model. The plots are given in Figure 8 , where the contour lines run from $-0.3 \mathrm{mV}$ to 0 $\mathrm{mV}$ in steps of 0.05 . The position of the ischaemic region can be seen in Figure 3. The jagged nature of the elevation in panel (i) in Figure 8, where $\mathrm{ISC}=90 \%$, is caused by the uneven epicardial surface above the ischaemic region which is at different heights above the top surface of the
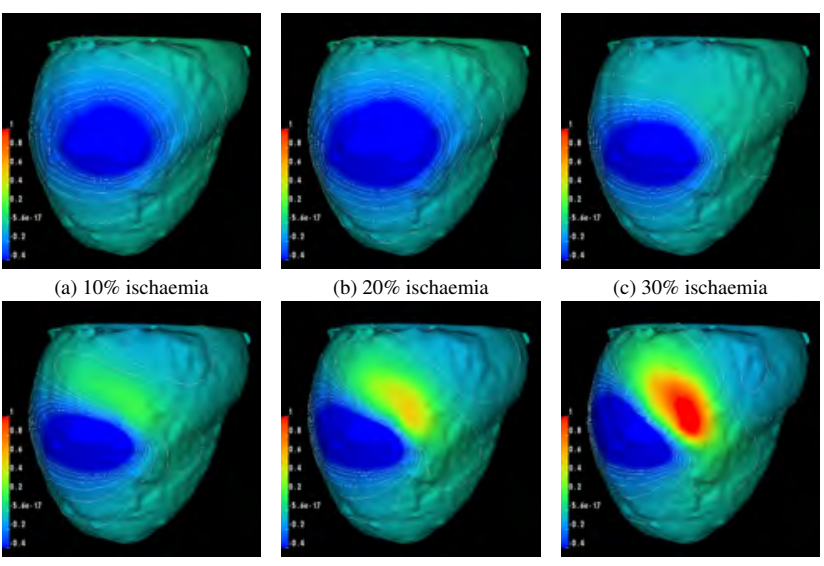

(b) $20 \%$ ischaemi
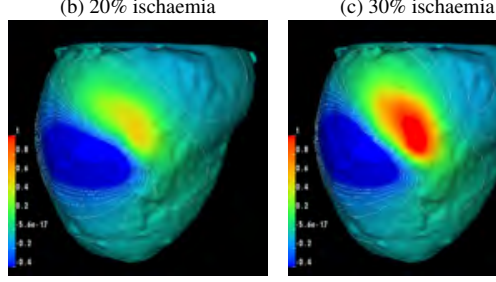

(d) $40 \%$ ischaemi

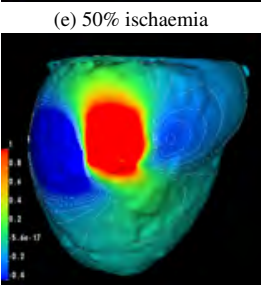

(h) $80 \%$ ischaemia

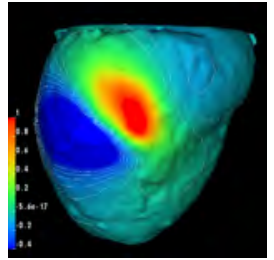

(f) $60 \%$ ischaemia

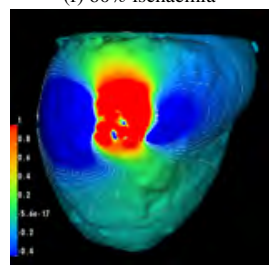

(g) $70 \%$ ischaemia

(i) $90 \%$ ischaemia

Figure 8: EPDs generated from mean values (Table 1), for the realistic model, for a range of ischaemic depths. Contour intervals are -0.3 to 0 in steps of 0.05 .

smoother ischaemic region, resulting in areas of lower and higher potential [19].

In a similar fashion to Figure 4, as ISC increases, the character of the EPD changes, from a single minimum over the ischaemic region (for example, Figure 8(a)) to a maximum over the ischaemic region flanked by two minima on opposite sides of the ischaemic region (for example, Figure $8(\mathrm{~g}))$. As in the half-ellipsoidal model, min2 does not appear until $\mathrm{ISC}=30 \%$. However, in this particular case of using the realistic model with mean values, we do not see the scenario of min1 plus max, but not min2 (i.e. EPD type 2).

Potential values for the EPD features are given in Table 5. Although values for $\mathrm{ISC}=90 \%$ are given for completeness, it is likely that the large values for $\max V$ are misleading due to the rough surface, discussed above, which may mean that the ischaemia is transmural at some points. The strength of the maximum increases as ISC increases, with values that are of the same order as those for the halfellipsoidal model, except for max $\mathrm{V}$ for the realistic model, which is much larger. The magnitudes of both min1V and min2V do not always increase with increasing ISC, unlike the monotonic increases as in the half-ellipsoidal case. However, for $\mathrm{ISC} \geq 60 \%$ both $\min 1 \mathrm{~V}$ and $\min 2 \mathrm{~V}$ do increase monotonically. In addition, the values for min $2 \mathrm{~V}$ in the realistic heart model are somewhat smaller than those in the half-ellipsoidal model.

A clockwise rotation of the pattern also occurs with 
Table 5: Potentials (in $\mathrm{mV}$ ) for various features of the EPDs in Figure 7, generated using mean values (Table 1), except for ISC, for the realistic model.

\begin{tabular}{c|ccc}
\hline $\begin{array}{c}\text { ISC } \\
\%\end{array}$ & $\min 1 \mathrm{~V}$ & $\operatorname{maxV}$ & $\min 2 \mathrm{~V}$ \\
\hline 10 & -0.66 & - & - \\
20 & -1.01 & - & - \\
30 & -0.88 & 0.01 & -0.07 \\
40 & -0.90 & 0.17 & -0.10 \\
50 & -0.90 & 0.54 & -0.10 \\
60 & -0.89 & 1.31 & -0.10 \\
70 & -1.06 & 2.67 & -0.14 \\
80 & -1.34 & 4.92 & -0.30 \\
90 & -2.04 & 14.3 & -0.65
\end{tabular}

increasing ISC, something that was also observed for the half-ellipsoidal model.

\subsubsection{Variation of EPDs with conductivity and ischaemic depth for the realistic model}

In this study, we changed some of the conductivities and examined the effect on the EPDs as ISC increased from $10 \%$ to $90 \%$ in the realistic model. We used two sets of extreme values (Table 1) for the longitudinal and normal conductivities (extremes 1: $g_{i l}=1.2, g_{e l}=3.6, g_{i n}=$ $0.05, g_{\text {en }}=1.5 \mathrm{mS} / \mathrm{cm}$ ) and (extremes $2: g_{i l}=3.6, g_{e l}=$ $\left.1.2, g_{\text {in }}=0.15, g_{\text {en }}=0.5 \mathrm{mS} / \mathrm{cm}\right)$ and means for the other parameters, except for ISC. These were chosen because, for the slab model [17], the intracellular and extracellular conductivities in a particular direction have opposite effects on the EPDs. We found that changing the conductivities does have an effect on the EPDs and the results are given in Figure 9 for extremes 1, Figure 10 for extremes 2, and Table 6 (for both).

We observe that, for both sets, the development of the EPD pattern, with increasing ISC, from min1 (only) to min1, max, min2, which includes a clockwise rotation of the features, is similar to the case where the mean conductivities were used (Section 3.2.1). However, the magnitudes of the potentials are either smaller (extremes 1 ) or larger (extremes 2) (Table 6). As in the mean case (Section 3.2.1), for both sets the strength of the maximum increases monotonically with ISC, while the mid-range ( $\mathrm{ISC}=30$ $60 \%$ ) values for both min $1 \mathrm{~V}$ and min2V stay fairly constant and the magnitudes of $\min 1 \mathrm{~V}$ and $\min 2 \mathrm{~V}$ for ISC > $60 \%$ increase with increasing ISC.

In addition, in this study, when $\mathrm{ISC}=30 \%$ for extremes 1 and $\mathrm{ISC}=40 \%$ for extremes 2 , we can identify a type 2 scenario, where we have min1 and $\max$, but not min2. The type 2 scenario was observed previously for the mean EPDs in the half-ellipsoidal model, but not for the mean EPDs

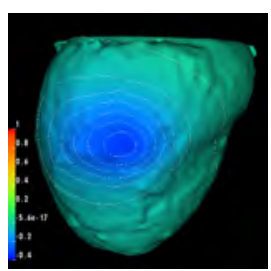

(a) $10 \%$ ischaemia

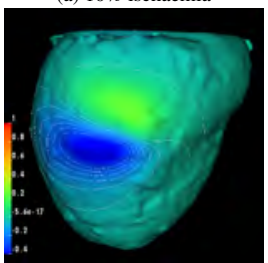

(d) $40 \%$ ischaemia

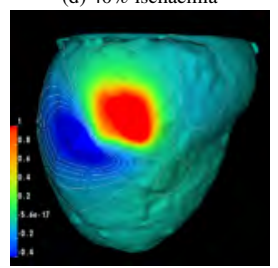

(g) $70 \%$ ischaemia

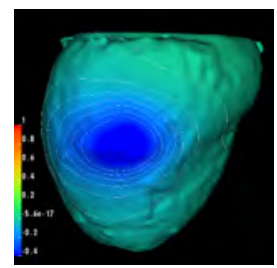

(b) $20 \%$ ischaemia

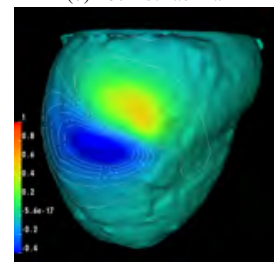

(e) $50 \%$ ischaemia

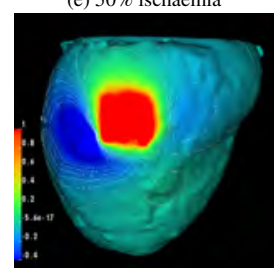

(h) $80 \%$ ischaemia

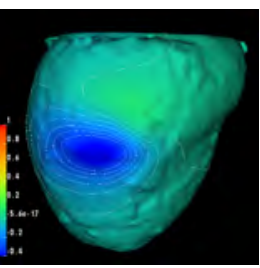

(c) $30 \%$ ischaemia

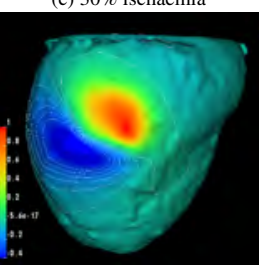

(f) $60 \%$ ischaemia

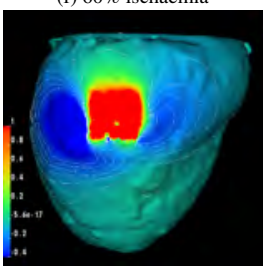

(i) $90 \%$ ischaemia
Figure 9: EPDs generated from extreme longitudinal and normal conductivities (extremes 1) (see Table 1 and text), using the realistic model, for a range of ischaemic depths. Contour intervals are -0.3 to 0 in steps of 0.05 .

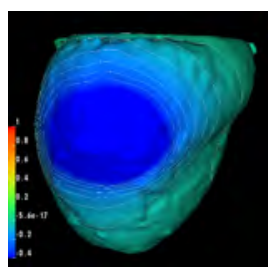

(a) $10 \%$ ischaemia

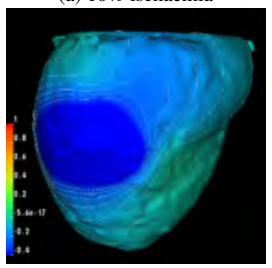

(d) $40 \%$ ischaemia

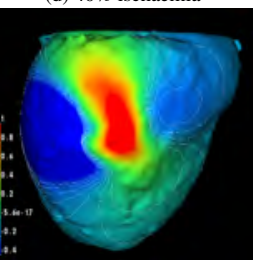

(g) $70 \%$ ischaemia

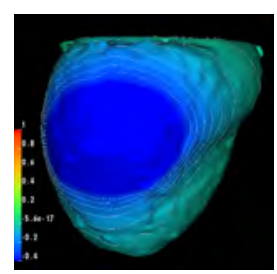

(b) $20 \%$ ischaemia

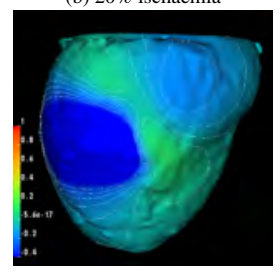

(e) $50 \%$ ischaemia

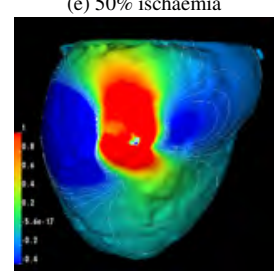

(h) $80 \%$ ischaemia

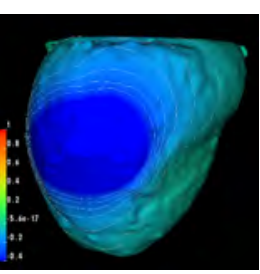

(c) $30 \%$ ischaemia

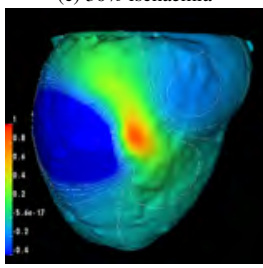

(f) $60 \%$ ischaemia

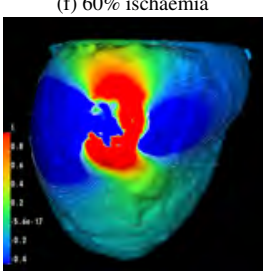

(i) $90 \%$ ischaemia
Figure 10: EPDs generated from extreme longitudinal and normal conductivities (extremes 2) (see Table 1 and text), using the realistic model, for a range of ischaemic depths. Contour intervals are -0.3 to 0 in steps of 0.05 . 
Table 6: Potentials (in $\mathrm{mV}$ ) for various features of EPDs generated using extreme (see text for details) longitudinal and normal conductivities. (Left) extremes 1 (Figure 9) and (right) extremes 2 (Figure $10)$.
We consider the effect of the inputs on EPDs of types 1-3 by producing design data of two types. For EPD types 1 and 2 we allow ISC to vary between $10 \%$ and $60 \%$ and for EPD type 3 ISC to vary between $10 \%$ and $90 \%$. All other inputs are allowed to vary across their full ranges (Table 1) and an LHC sampling routine is used to produce sets of nine inputs (Section 2.5).

\begin{tabular}{|c|c|c|c|c|c|c|}
\hline \multirow[b]{2}{*}{$\begin{array}{c}\text { ISC } \\
\%\end{array}$} & \multicolumn{3}{|c|}{ Extremes 1} & \multicolumn{3}{|c|}{ Extremes 2} \\
\hline & $\min 1 \mathrm{~V}$ & $\operatorname{maxV}$ & $\min 2 \mathrm{~V}$ & $\min 1 \mathrm{~V}$ & $\operatorname{maxV}$ & $\min 2$ \\
\hline 10 & -0.33 & - & - & .97 & - & - \\
\hline 20 & & - & - & 1.54 & - & - \\
\hline 30 & -0.44 & 0.08 & - & -1.39 & - & - \\
\hline 40 & -0.46 & 0. & -0.05 & -1.37 & 0.02 & - \\
\hline 50 & -0.49 & 0.52 & -0.06 & -1.32 & 0.11 & -0.18 \\
\hline 60 & -0.49 & 1.00 & -0.05 & -1.26 & 0.87 & -0.18 \\
\hline 70 & 56 & 1. & -0.06 & -1.45 & 2.2 & -0.23 \\
\hline 80 & 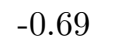 & 3. & -0 . & -1.80 & 4.96 & -0.45 \\
\hline 90 & -0.86 & 8.28 & -0.28 & -4.37 & 19.56 & -1.23 \\
\hline
\end{tabular}

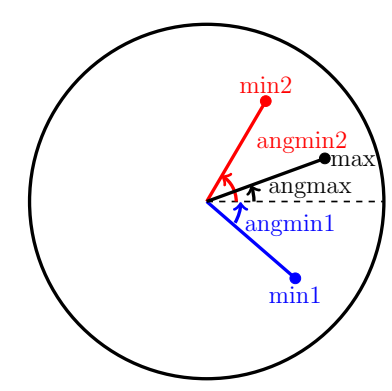

Figure 11: The angles angmin1, angmax and angmin2 are illustrated for an example of the EPD polar plot features $\min 1, \max , \min 2$.

for the realistic model (Section 3.2.1). In addition, we see that min1 does not appear until ISC is $40 \%$ (extremes 1) or $50 \%$ (extremes 2), which is later than the mean case for both models (Tables 2 and 5).

The above comparisons between the two models show that the half-ellipsoidal model produces EPDs that are reasonably similar to those in the realistic model. So, in order to study the effect of changes of fibre rotation, which is not possible in the realistic model, combined with changes in conductivities and ISC, we now return to the half-ellipsoidal model and investigate these various effects in more generality.

\subsection{Sensitivities of outputs to uncertainties in nine inputs in the half-ellipsoidal model}

In this section we consider the effect of varying all nine inputs from Table 1, that is, ROT, ISC, $g_{b}$ and $g_{p q}(p=$ $i, e, q=l, t, n)$, on the features $\min 1, \max$ and $\min 2$ of the EPDs. For each of these features we consider outputs that consist of their potentials, $\min 1 \mathrm{~V}, \max \mathrm{V}$ and $\min 2 \mathrm{~V}$, respectively, as well as their angles, angmin1, angmax and angmin2, respectively. The angles are defined to be those the feature makes in the polar plot (e.g. Figure 4(i)) with the horizontal axis as shown in Figure 11.
The rationale behind the first set of design data is to keep ISC reasonably low, but still in as wide a range as possible, so that sufficient EPDs of types 1 and 2 are produced (out of 1000 sets of inputs, 160 were of type 1 and 195 of type 2). Since EPDs of type 3 occur over nearly the full range of ischaemic depths, in the second set it was possible to vary ISC from $10 \%$ to $90 \%$ and obtain 320 type 3 EPDs from 400 sets of inputs.

In fact, during this study, we found that type 1 EPDs can occur for ISC from 10-35\%, type 2 for ISC in the range $10-46 \%$ and type 3 for ISC $>14 \%$, which may have implications for diagnosis (see Discussion).

We examined the sensitivities of the outputs to the inputs using both GP emulators (Section 2.5.1) and PLS regression (Section 2.5.2). For each method, we fitted emulators for each of the relevant outputs for that EPD, bearing in mind that for type 1 EPDs, for example, the only feature that occurs is min1. An example of the design data for $\operatorname{maxV}$ and angmax, for type 2 EPDs, can be found in Figure 12 and the remainder of the design data is available in the Supplementary material.

We then used the GP emulators to produce main effect plots, as described in Section 2.5.1. Examples of these plots for EPD type 2 maxV and angmax are given in Figure 13. In each case 0 on the vertical scale represents the emulator mean value for that output $(0.22 \mathrm{mV}$ for $\max \mathrm{V}$ and $15.6^{\circ}$ for angmax). These plots indicate that the main inputs to which EPD type $2 \mathrm{maxV}$ and angmax are sensitive are ISC and $g_{e n}$, in both cases, although the relationship is positive for maxV and negative for angmax.

Next we calculated sensitivity indices (Section 2.5.1) for each GP emulator using means of 0.5 and variances of 0.2 for each input variable. These indices quantify the contribution of the variance in each input to the variance in each output. The sensitivity indices for the GP emulator are given in Table 7 in the rows designated GP. Also given is the emulator expectation of the mean of each output $E^{*} E\{f(x)\}$. Values in bold indicate those inputs to which the output is particularly sensitive. We see that the results for EPD type $2 \operatorname{maxV}$ and angmax are consistent with the main effect plots in Figure 13, allowing for the unsigned nature of the GP sensitivity values, since they are calculated using variances (Equation (6)).

We also analysed the design data using a PLS approach (Section 2.5.2) to produce regression coefficients that indi- 

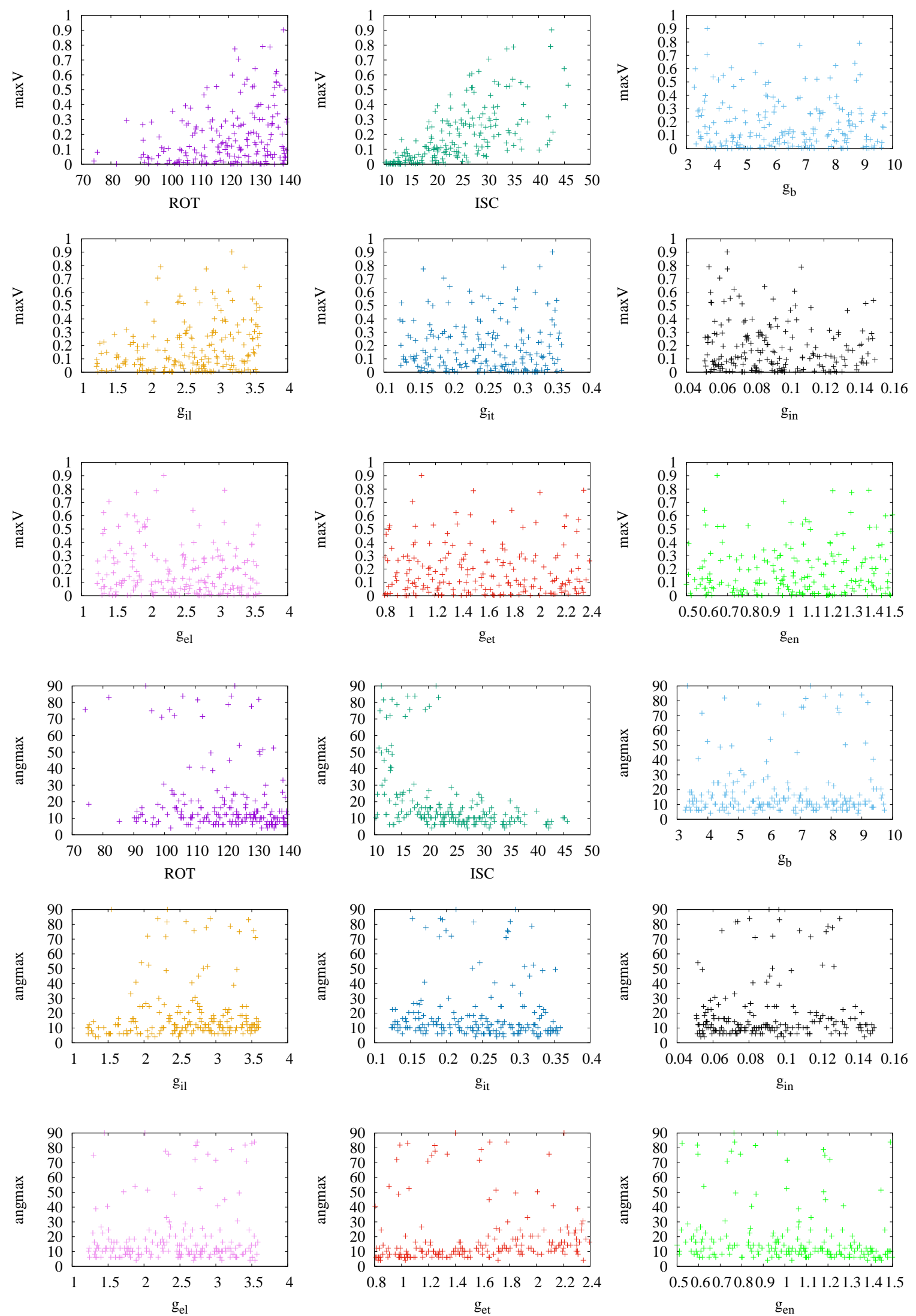

Figure 12: Design data for EPD type 2 (top half of figure) $\operatorname{maxV}$ (in $\mathrm{mV}$ ) and (bottom half of figure) angmax (in ${ }^{\circ}$ ). 

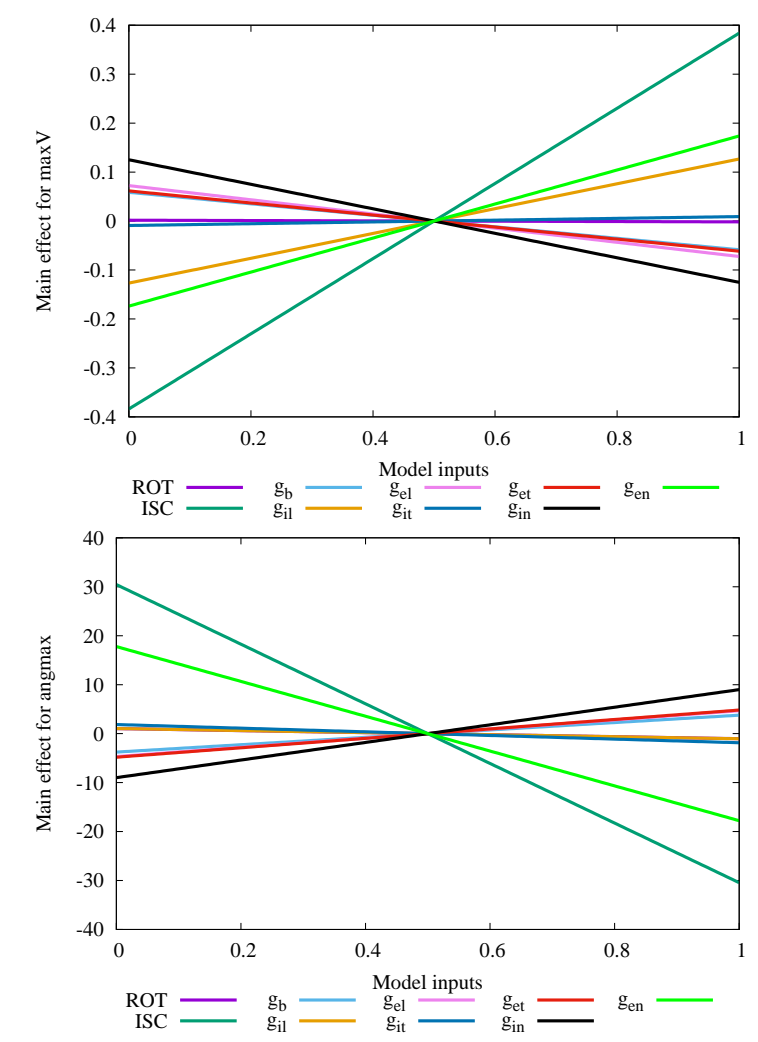

Figure 13: Main effects plots for maxV (top panel) and angmax (bottom panel) for type 2 EPDs

cate the direction and magnitude of change for each output, due to each input. These coefficients are also in Table 7 in the rows labelled PLS. Note that the PLS coefficients are signed, unlike the GP sensitivities. Ignoring the sign of the values, we see that the GP sensitivities and the PLS regression coefficients consistently identify the same inputs as the ones to which the outputs are sensitive. Note that the magnitude of the coefficients is relevant only within each row and should not be compared between methods.

We repeated (Section 3.1.2) these two studies with a wider border zone and we also investigated the effect of using a larger ischaemic region and the effect of moving the ischaemic region up and down the ventricle. We also examined the effect of using a 'new' representation for the transmembrane potential (equation (4)). We found that, although the values for the potentials were sometimes different, this made no difference to determining the inputs to which various outputs were sensitive, except in two borderline cases $\left(g_{e l}\right.$ for type $2 \min 1 \mathrm{~V}$ and ISC for type 3 min1V). We made this clear by marking in bold in Table 7 only those inputs to which an output is sensitive for all the scenarios considered. A summary of these effects is given in Table 8, where the black arrows indicate significant sensitivity (including its direction) and blanks represent little or no sensitivity.

The results in Tables 7 and 8 indicate that all outputs are sensitive to ROT and/or ISC and a few are moderately sensitive to conductivities such as $g_{i l}, g_{e l}$ and $g_{e n}$ and this is consistent between the two methods. The indices show that both min1V and angmin1 are sensitive to ROT, for all EPD types. Increasing ROT results in increases in the magnitude of min1V and in angmin1 (i.e. min1 moves anticlockwise).

On the other hand, increasing ISC has a clockwise effect on $\min 1$ (this is found for types 1 and 2 and is also found for type 3 for the wider ischaemic border - this is the only case where there is a difference between the results of the narrow and wide borders), but ISC has no effect on the magnitude of min1 for EPD types 1 and 2 .

However, ISC does affect the magnitude and position of max and min2, with increasing ISC increasing the magnitude of $\max \mathrm{V}$ and $\min 2 \mathrm{~V}$ and having a clockwise effect on their position. These results are consistent with what was shown in Section 3.1; although, in that case it was just for EPDs calculated with mean values.

The main results can be summarised as follows:

- the outputs are not sensitive to $g_{b}, g_{i t}$ and $g_{e t}$.

- min1 is sensitive to ROT (increasing magnitude and anticlockwise rotation for increasing ROT) and ISC (clockwise rotation for increasing ISC).

- $\max$ is sensitive to ISC (increasing magnitude and clockwise rotation for increasing ISC).

- $\min 2$ is sensitive to ISC (increasing magnitude and clockwise rotation for increasing ISC).

\subsection{Sensitivities of outputs to uncertainties in bidomain conductivities (only) in the half-ellipsoidal model}

Since the effects of ROT and ISC on the outputs seems to be so strong (Table 7), in comparsion with the other inputs, and the effect of $g_{b}$ is minimal, we decided to fix $\mathrm{ROT}=100^{\circ}$ and $g_{b}=6.5 \mathrm{mS} / \mathrm{cm}$ (their mean values in Table 1 ) to allow us to investigate the relative effect of the bidomain conductivities $\left(g_{p q}, p=i, e, q=l, t, n\right)$. New design data were produced using ISC $=10 \%$ to get types 1 and 2 EPDs and $\mathrm{ISC}=60 \%$ to get type 3 EPDs. In the first case, 500 initial sets resulted in 312 type 1, 188 type 2 sets and 0 type 3 sets and in the second case the 250 inputs produced only type 3 outputs. This is consistent with the ISC ranges where the various EPD types occur, as listed in Section 3.3.

As in Section 3.3, emulators were fitted for each output and each type of EPD, and PLS regression coefficients were produced. The results, given in Table 9 as well as in Table 8 via the red arrows, indicate that the outputs are sensitive to the longitudinal and normal bidomain conductivities, but not to the transverse ones. In particular, there is a clockwise relationship between $g_{i l}$ and angmin 1 for all EPD types, whereas for $g_{\text {in }}$ the effect is anticlockwise. In addition, $g_{e l}$ has a positive effect on min1V, again for all EPD types, and in this case, $g_{i l}$ has the opposite effect. This suggests that it might be worthwhile to look at the sensitivity of EPD outputs to ratios of longitudinal and normal conductivities. 
Table 7: Emulator expected means $E^{*} E\{f(x)\}$ (in $\mathrm{mV}$ for potentials and ${ }^{\circ}$ for angles) and sensitivities of the EPD feature outputs in the half-ellipsoidal model to nine inputs (Table 1), calculated as GP emulator sensitivities (GP) or by partial least squares (PLS). PLS sensitivities are signed, GP sensitivities are not. Values in bold indicate those inputs to which the output is particularly sensitive.

\begin{tabular}{|c|c|c|c|c|c|c|c|c|c|c|c|c|}
\hline Feature & $\begin{array}{l}\text { EPD } \\
\text { Type }\end{array}$ & Method & $E^{*} E\{f(x)\}$ & $\mathrm{ROT}$ & ISC & $g_{b}$ & $g_{i l}$ & $g_{e l}$ & $g_{i t}$ & $g_{\text {et }}$ & $g_{\text {in }}$ & $g_{\text {en }}$ \\
\hline \multirow[t]{2}{*}{$\min 1 \mathrm{~V}$} & \multirow[t]{4}{*}{1} & PLS & & -0.76 & -0.01 & -0.13 & -0.16 & 0.28 & 0.04 & 0.19 & -0.29 & 0.07 \\
\hline & & GP & -0.47 & 0.54 & 0.00 & 0.01 & 0.02 & 0.07 & 0.00 & 0.03 & 0.08 & 0.01 \\
\hline \multirow[t]{2}{*}{ angmin1 } & & PLS & & 0.59 & -0.65 & 0.18 & -0.49 & 0.03 & 0.10 & -0.06 & 0.32 & -0.18 \\
\hline & & GP & -25.6 & 0.18 & 0.36 & 0.02 & 0.11 & 0.00 & 0.01 & 0.00 & 0.05 & 0.02 \\
\hline \multirow[t]{2}{*}{$\min 1 \mathrm{~V}$} & \multirow[t]{4}{*}{2} & PLS & & -0.57 & -0.24 & 0.00 & -0.47 & 0.43 & 0.04 & 0.29 & -0.10 & -0.12 \\
\hline & & GP & -0.59 & 0.41 & 0.06 & 0.00 & 0.18 & 0.13 & 0.00 & 0.06 & 0.01 & 0.01 \\
\hline \multirow[t]{2}{*}{ angmin1 } & & PLS & & 0.95 & -0.65 & 0.11 & -0.20 & -0.03 & 0.06 & -0.09 & 0.08 & 0.07 \\
\hline & & GP & -22.6 & 0.65 & 0.28 & 0.00 & 0.02 & 0.00 & 0.00 & 0.00 & 0.00 & 0.00 \\
\hline \multirow[t]{2}{*}{$\min 1 \mathrm{~V}$} & \multirow[t]{4}{*}{3} & PLS & & -0.48 & -0.51 & -0.10 & -0.24 & 0.39 & 0.01 & 0.27 & -0.06 & -0.28 \\
\hline & & GP & -0.65 & 0.19 & 0.25 & 0.01 & 0.04 & 0.12 & 0.00 & 0.05 & 0.00 & 0.06 \\
\hline \multirow[t]{2}{*}{ angmin1 } & & PLS & & 0.76 & -0.32 & 0.13 & -0.31 & -0.05 & 0.03 & -0.22 & 0.14 & 0.33 \\
\hline & & GP & -30.1 & 0.38 & 0.08 & 0.01 & 0.06 & 0.00 & 0.00 & 0.03 & 0.01 & 0.06 \\
\hline \multirow[t]{2}{*}{$\max V$} & \multirow[t]{4}{*}{2} & PLS & & 0.00 & 0.98 & -0.19 & 0.37 & -0.23 & 0.03 & -0.19 & -0.38 & 0.53 \\
\hline & & GP & 0.22 & 0.00 & 0.53 & 0.01 & 0.06 & 0.02 & 0.00 & 0.01 & 0.06 & 0.11 \\
\hline \multirow[t]{2}{*}{ angmax } & & PLS & & -0.02 & -0.73 & 0.11 & -0.03 & 0.14 & -0.05 & 0.14 & 0.25 & -0.50 \\
\hline & & GP & 15.6 & 0.00 & 0.38 & 0.01 & 0.00 & 0.01 & 0.00 & 0.01 & 0.03 & 0.13 \\
\hline \multirow[t]{2}{*}{$\max V$} & \multirow[t]{4}{*}{3} & PLS & & 0.04 & 0.86 & -0.04 & 0.28 & -0.20 & 0.01 & -0.13 & -0.17 & 0.23 \\
\hline & & GP & 1.62 & 0.00 & 0.58 & 0.00 & 0.05 & 0.03 & 0.00 & 0.01 & 0.02 & 0.03 \\
\hline \multirow[t]{2}{*}{ angmax } & & PLS & & 0.33 & -0.96 & -0.01 & 0.06 & 0.04 & -0.04 & 0.03 & 0.00 & -0.10 \\
\hline & & GP & 2.9 & 0.06 & 0.59 & 0.00 & 0.00 & 0.00 & 0.00 & 0.00 & 0.00 & 0.01 \\
\hline \multirow[t]{2}{*}{$\min 2 \mathrm{~V}$} & \multirow[t]{4}{*}{3} & PLS & & 0.28 & -0.92 & -0.05 & -0.15 & 0.17 & 0.07 & -0.03 & -0.01 & -0.09 \\
\hline & & GP & -0.41 & 0.04 & 0.52 & 0.00 & 0.01 & 0.01 & 0.00 & 0.00 & 0.00 & 0.00 \\
\hline \multirow[t]{2}{*}{ angmin2 } & & PLS & & 0.14 & -0.88 & -0.07 & 0.27 & 0.14 & 0.09 & -0.03 & -0.21 & -0.17 \\
\hline & & GP & 42.7 & 0.01 & 0.49 & 0.00 & 0.04 & 0.01 & 0.00 & 0.01 & 0.02 & 0.01 \\
\hline
\end{tabular}

Table 8: Summary of the outputs (rows) and the input variables (columns) to which they are sensitive, for the half-ellipsoidal model. Blank spaces indicate no significant relationship. An increase in the input variable that results in an increase in the output is represented by an upward pointing arrow and an increase in the input variable that results in a decrease in the output is represented by a downward pointing arrow. Black arrows are for results from Section 3.3 and red arrows from Section 3.4.

\begin{tabular}{lc|ccccccccc}
\hline Feature & $\begin{array}{c}\text { EPD } \\
\text { Type }\end{array}$ & ROT & ISC & $g_{b}$ & $g_{i l}$ & $g_{\text {el }}$ & $g_{i t}$ & $g_{\text {et }}$ & $g_{\text {in }}$ & $g_{\text {en }}$ \\
\hline min1V & 1 & $\downarrow$ & & & & $\uparrow$ & & & $\downarrow$ & \\
angmin 1 & & $\uparrow$ & $\downarrow$ & & $\downarrow$ & & & & $\uparrow$ & \\
$\min 1 \mathrm{~V}$ & 2 & $\downarrow$ & & & $\downarrow \downarrow$ & $\uparrow$ & & & & \\
angmin 1 & & $\uparrow$ & $\downarrow$ & $\downarrow$ & & & $\uparrow$ & \\
$\min 1 \mathrm{~V}$ & 3 & $\downarrow$ & & $\downarrow$ & $\uparrow \uparrow$ & $\uparrow$ & & $\downarrow$ \\
$\operatorname{angmin} 1$ & & $\uparrow$ & & $\downarrow$ & $\downarrow$ & & $\downarrow$ & & $\uparrow$ \\
\hline $\operatorname{maxV}$ & 2 & & $\uparrow$ & $\uparrow$ & & & $\downarrow$ & $\uparrow \uparrow$ \\
$\operatorname{angmax}$ & & & $\downarrow$ & $\downarrow$ & & & $\uparrow$ & $\uparrow$ & $\downarrow \downarrow$ \\
$\max$ & 3 & & $\uparrow$ & $\uparrow$ & $\downarrow$ & & & $\uparrow$ \\
$\operatorname{angmax}$ & & & $\downarrow$ & $\uparrow$ & & & & & $\downarrow$ \\
\hline $\min 2 \mathrm{~V}$ & 3 & & $\downarrow$ & & & $\uparrow$ & & & & $\downarrow$ \\
$\operatorname{angmin} 2$ & & & $\downarrow$ & $\uparrow$ & & & & $\downarrow$ & $\uparrow$
\end{tabular}


Table 9: PLS sensitivities of EPD outputs in the half-ellipsoidal model to the six bidomain conductivities, produced using mean values (Table 1) for ROT and $g_{b}$. Values in bold indicate those inputs to which the output is particularly sensitive.

\begin{tabular}{lc|cccccc}
\hline Feature & $\begin{array}{c}\text { EPD } \\
\text { Type }\end{array}$ & $g_{i l}$ & $g_{e l}$ & $g_{i t}$ & $g_{\text {et }}$ & $g_{i n}$ & $g_{\text {en }}$ \\
\hline min1V & 1 & -0.32 & $\mathbf{0 . 5 9}$ & 0.04 & 0.39 & $\mathbf{- 0 . 5 3}$ & 0.10 \\
angmin1 & & $\mathbf{- 0 . 9 2}$ & 0.00 & 0.17 & -0.06 & $\mathbf{0 . 5 7}$ & -0.28 \\
min1V & 2 & $\mathbf{- 0 . 4 8}$ & $\mathbf{0 . 4 9}$ & 0.04 & 0.40 & -0.35 & -0.04 \\
angmin1 & & $\mathbf{- 0 . 8 3}$ & -0.19 & 0.23 & -0.22 & $\mathbf{0 . 5 9}$ & -0.02 \\
min1V & 3 & $\mathbf{- 0 . 4 2}$ & $\mathbf{0 . 6 4}$ & 0.06 & $\mathbf{0 . 4 3}$ & -0.09 & $\mathbf{- 0 . 4 4}$ \\
angmin1 & & $\mathbf{- 0 . 4 9}$ & -0.12 & 0.02 & $\mathbf{- 0 . 4 6}$ & 0.23 & $\mathbf{0 . 6 2}$ \\
\hline maxV & 2 & $\mathbf{0 . 8 0}$ & -0.36 & -0.07 & -0.29 & $\mathbf{- 0 . 9 4}$ & $\mathbf{0 . 7 5}$ \\
angmax & & $\mathbf{- 0 . 6 6}$ & 0.09 & -0.07 & $\mathbf{0 . 4 9}$ & $\mathbf{0 . 9 7}$ & $\mathbf{- 0 . 8 7}$ \\
maxV & 3 & $\mathbf{0 . 5 3}$ & $\mathbf{- 0 . 4 4}$ & 0.03 & -0.26 & -0.28 & $\mathbf{0 . 5 6}$ \\
angmax & & $\mathbf{0 . 4 6}$ & 0.28 & -0.22 & -0.07 & 0.11 & $\mathbf{- 0 . 4 6}$ \\
\hline min2V & 3 & -0.29 & $\mathbf{0 . 7 6}$ & 0.25 & 0.07 & -0.27 & $\mathbf{- 0 . 4 3}$ \\
angmin2 & & $\mathbf{0 . 5 5}$ & 0.40 & 0.17 & -0.13 & $\mathbf{- 0 . 4 4}$ & $\mathbf{0 . 5 5}$
\end{tabular}

\subsection{Sensitivities of outputs to ratios of bidomain conduc- tivities (only) in the half-ellipsoid model}

We used the same design data as in Section 3.4, but this time we looked at the sensitivity of the outputs, using PLS regression, to ratios of the conductivities $g_{i q} / g_{e q}(q=$ $l, t, n)$, and $g_{i l} / g_{i t}, g_{i l} / g_{i n}, g_{e l} / g_{e t}$ and $g_{e l} / g_{e n}$. We found (not presented) that a few outputs were sensitive to either $g_{i n} / g_{\text {en }}$ or $g_{i l} / g_{i n}$, but that the most significant ratio was $g_{e l} / g_{e n}$. The $g_{e l} / g_{e n}$ ratio was positively correlated with $\min 1 \mathrm{~V}$, for all EPD types, negatively with $\max \mathrm{V}$, and positively with min2V. It was also positively correlated with angmax and angmin2, and negatively with angmin1 for EPD type 3.

\section{Discussion}

One of the most significant findings of this study is the change in the form of the EPD with ischaemic depth. The ST segment EPDs have been characterised here as consisting of three types that develop as ISC increases: type 1 (min1 only, located over the ischaemic region), type 2 (max over the ischaemic region, plus min1 over a boundary) and type 3 (max over the ischaemic region, plus min1 and min2 over opposite boundaries).

A similar progression was identified in previous work [17] in a slab model, with ST depression (type 1) and ST elevation being very similar to types 1 and 3 , respectively (apart from the asymmetry here in min1 and min2). The second case for the slab model consisted of a tripole of three minima, where the central minimum was the largest (i.e. closest to zero), whereas type 2 for both the halfellipsoidal model and the realistic model was just max plus min1. Other studies [9, 7, 3], using realistic geometries, have also identified the type 1 and type 3 scenarios found in this study, but not the type 2 case. This is not surprising as the ISC values of around $20 \%-30 \%$ where this seems to occur were not presented, although $\mathrm{ISC}=25 \%$ was described [7] as a 'hybrid' of low $(<10 \%)$ and medium (30\%-50\%) ischaemia.

The exact position of min1 in relation to the ischaemic region, in the type 1 case, is not entirely clear. It appears to be either partially or fully over the ischaemic region (see for example, Figures 8-10(a)-(c) and Figure 4(a)). Its position depends on the value of ISC, as that affects the development of max and the movement of min1 to the boundary of the ischaemic region. This is confirmed by the correlation of ISC with angmin1 for EPD types 1 and 2 as well as with angmax for EPD type 2 (Table 7). This would appear to make locating the ischaemic region difficult when ISC is low.

Another aspect of this change in EPD with ISC is the asynchronous development of min1 and min2, found for both the half-ellipsoidal and realistic models. Min2 appears later than min1 but may then increase more rapidly so that for high values of ISC it can be stronger in magnitude than min1 for the half-ellipsoidal model (e.g. Figure 4(g)-(i), Table 2). To the best of our knowledge this has not been mentioned explicitly in previous work; however, two unequal minimums are shown in [3] (Figure 1B) and [9] (Figure 2) and it is hinted at in the latter work when it was stated that 'ST depression along at least one side of the ischemic patch increased with the degree of transmural ischaemia'. The fact that min1 and min2 did not develop independently in the slab model [17] is to be expected due to the symmetry of the model.

The present study found, for the half-ellipsoidal model, that the min 1 only case (type 1 EPD), can occur for ISC up to $35 \%$ (Section 3.3). Also an example of a type $1 \mathrm{EPD}$ was found for $\mathrm{ISC}=40 \%$ for the realistic model (Figure $10(d))$. This is consistent with work for the slab model [17] and modelling work by Potse et al. [3], which found that min1 only EPDs can occur over a much wider range than previously thought (up to $\mathrm{ISC}=30 \%-40 \%$ ). 
Similarly the min1 plus max case (type 2 EPDs) was found here to occur for ISC up to $46 \%$ for the half-ellipsoida model (Section 3.3) and in this case min1 has moved to the boundary between the healthy and ischaemic tissue. This is consistent with the conclusion of Hopenfeld et al. [7] that for ISC $\geq 40 \%$ there is at least one area of ST depression located at that boundary (i.e. min1 for type 2 EPD or min1 and min2 for type 3 EPD).

The sensitivity study to the nine inputs (Tables 7 and 8 ) found that the magnitudes of $\max \mathrm{V}$ and $\min 2 \mathrm{~V}$ increase with increasing ISC, whereas this is not the case for min1V, except for EPD type 3. This is consistent with the results for the realistic model (Table 6) and with previous work [9] that found increasing magnitudes for the maximum and at least one of the minima with increasing ISC. Our sensitivity study also indicated that none of the EPD features is sensitive to the blood conductivity or transverse conductivity values, which agrees with the findings a similar study using a slab model [17].

The connection between increasing ISC and a clockwise rotation of the EPD features was shown for the realistic model's three sets of plots (Figures 8-10) and more systematically for the half-ellipsoidal model (Table 8) and all of the EPD features. This is consistent with similar systematic work using the slab model [17] and earlier work [9, 30] considering a few scenarios in a canine heart. Previous work $[9,19]$ has also identified the anticlockwise effect of increasing fibre rotation on the form of the EPD, again for a limited number of scenarios, and the systematic work done here is in agreement with that, but only for min1 (Table 8).

Putting all this together might suggest applying the following diagnostic criteria to ST segment EPDs: a single minimum (regardless of whether a maximum is observed) indicates a low level of subendocardial ischaemia; on the other hand, two minima (both of which are not near zero) indicates a high level of subendocardial ischaemia. In the latter case, the ischaemic region may be able to be located in a similar fashion to transmural ischaemia, by considering the position of the maximum, which should be above the ischaemic region, combined with the position of the two minima, which should be along the borders of the ischaemic region. This is consistent with the experimental study of Li et al. [5], which found depression over the lateral borders of the partial thickness ischaemic region.

Based on the sensitivity studies carried out in this work, we conclude that the effect of conductivity values on the maximum and minima is minor, in comparison with the effect of ischaemic depth and fibre rotation (Section 3.3 and Table 7 ). We also note that fibre rotation does not affect max or min2, and while it affects the value and position of min1, it does not affect its existence (Table 8), so the above conclusions are expected to apply in spite of the intrinsic variability that is found across the human population.

However, experimental studies have shown [2] that the situation is not so clear-cut as the above discussion might imply. Cases that may cause difficulty are where the magnitude of min2 is small compared with min1 despite ISC being quite high. Here this occurs for the 'mean' realistic model (Table 5) but not the 'mean' half-ellipsoidal model (Table 2). The difference in the two models may be related to differences in fibre rotation, since the value at which min2 appears is higher for higher ROT, while, for fixed ISC, increasing ROT increases the magnitude of min1 but decreases the magnitude of min2 (Section 3.1.3). In addition, the above study [2] has demonstrated that ischaemia that is not transmural, especially when the ST segment is used for identification, is often not detected even on the epicardial surface. The authors suggest that this may be due to signal attenuation, masking from other ischaemic sources or electrical anisotropy. This is in addition to the fact that these results on the epicardium may not translate directly to the body surface and therefore to the ECG.

Although we have shown that, for the half-ellipsoidal model, the size and position (excluding near to the apex and base of the ventricle) of the ischaemic region, the width of the border zone between the healthy and ischaemic tissue, and a parabolic represenation of the transmembrane potential, have little effect on the outputs to which the inputs are sensitive, there still remain a number of potential limitations in this study.

These include the shape of the ischaemic region, since previous work [19], which compared rectangular, cylindrical and semi-ellipsoidal ischaemic regions in the same halfellipsoidal model used in this work, using Clerc's [31] conductivities, found that the EPDs produced by the rectangular and cylindrical regions were very similar, whereas the effect of the semi-ellipsoidal region was that min2 did not appear until ISC was larger. Further research is necessary to determine whether this is likely to have any effect on the sensitivity of the outputs to the various inputs.

In an effort to match experimental studies [21], in this work we have considered one particular new representation for the transmembrane potential (Section 2.2), but this is something that could be the subject of further research [32], along with an examination of the effect of considering partial thickness ischaemia that is not subendocardial. This is motivated by the findings of a study by Aras et al. [2] that acute ischaemia arises throughout the ventricular wall, rather than being limited to the subendocardium. Another possible avenue of research involves investigating the effect of using different conductivity values in the ischaemic and healthy regions. Finally, in order to connect this work with changes in the ECG, it will be necessary use a coupled realistic heart model and a torso model.

\section{Conclusion}

Using both a half-ellipsoidal and a realistic model of the left ventricle, this work examined the development of ST segment depression and elevation in the EPD, as subendocardial ischaemic depth was increased. In addition, inputs 
to the model were systematically varied and it was determined that the EPD features were most sensitive to fibre rotation and ischaemic depth.

Three possible EPD patterns were identified: a single minimum that is located near or over the ischaemic region; a maximum over the ischaemic region plus this minimum near the boundary; and finally, this maximum with two minimums at opposite sides on the ischaemic border. Using a wide range of inputs, it was found that the single minimum scenario could be found for ischaemic depths of up to around $35 \%$, and the maximum plus one minimum case up to around $45 \%$. This may partially explain the difficulties that occur when locating the position of low to medium ischaemia, since the position of the single minimum changes from over the ischaemic region to its border.

\section{Acknowledgements}

The authors would like to acknowledge Josef Barnes for his development of the realistic ventricular model that is used in this work.

\section{References}

[1] S. Shome, J. G. Stinstra, B. Hopenfeld, B. B. Punske, R. S. MacLeod, A study of the dynamics of cardiac ischaemia using experimental and modeling approaches, in: Proceedings of the IEEE Engineering in Medicine and Biology 26th Annual International Conference, IEEE EMBS, IEEE Press, 2004, pp. 3585-3588.

[2] K. Aras, B. Burton, D. Swenson, R. MacLeod, Spatial organisation of acute myocardial ischaemia, Journal of Electrocardiology 49 (2016) 323-336.

[3] M. Potse, R. Coronel, S. Falcao, A. R. LeBlanc, A. Vinet, The effect of lesion size and tissue remodeling on ST deviation in partial-thickness ischemia;, Heart Rhythm 4 (2) (2007) 200-206.

[4] R. A. Guyton, J. H. McClethan, G. E. Newman, L. L. Michaelis, Significance of subendocardial S-T segment elevation caused by coronary stenosis in the dog, American Journal of Cardiology 40 (1977) 373-380.

[5] D. Li, C. Y. Li, A. C. Yong, D. Kilpatrick, Source of electrocardiographic ST changes in subendocardial ischemia, Circulation Research 82 (1998) 957-970.

[6] L. Tung, A bi-domain model for describing ischaemic myocardial D-C potentials, Ph.D. thesis, Massachusetts Institute of Technology (June 1978).

[7] B. Hopenfeld, J. G. Stinstra, R. S. MacLeod, The effect of conductivity on ST-segment epicardial potentials arising from subendocardial ischemia, Annals of Biomedical Engineering 33 (6) (2005) 751-763.

[8] M. Potse, A.-R. LeBlanc, R. Cardinal, A. Vinet, St elevation or depression in subendocardial ischemia?, in: 28th IEEE EMBS Annual International Conference, 2006, pp. 3899-3902.

[9] B. Hopenfeld, J. G. Stinstra, R. S. MacLeod, Mechanism for ST depression associated with contiguous subendocardial ischaemia, Journal of Cardiovascular Electrophysiology 15 (2004) 1200-1206.

[10] P. R. Johnston, D. Kilpatrick, C. Y. Li, The importance of anisotropy in modelling ST segment shift in subendocardial ischaemia, IEEE Transactions on Biomedical Engineering 48 (12) (2001) 1366-1376.

[11] P. R. Johnston, D. Kilpatrick, The effect of conductivity values on ST segment shift in subendocardial ischaemia, IEEE Transactions on Biomedical Engineering 50 (2) (2003) 150-158.
[12] P. R. Johnston, Cardiac conductivity values - a challenge for experimentalists?, Noninvasive Functional Source Imaging of the Brain and Heart \& 2011 8th International Conference on Bioelectromagnetism (NFSI \& ICBEM) (2011) 39-43.

[13] B. M. Johnston, P. R. Johnston, The sensitivity of the passive bidomain equation to variations in six conductivity values, in: A. Boccaccini (Ed.), Proceedings of the 10th IASTED International Conference Biomedical Engineering (BioMed 2013), IASTED, ACTA Press, Calgary, Alberta, Canada, 2013, pp. $538-545$.

[14] B. J. Roth, Electrical conductivity values used with the bidomain model of cardiac tissue, IEEE Transactions on Biomedical Engineering 44 (4) (1997) 326-328.

[15] D. A. Hooks, M. L. Trew, B. J. Caldwell, G. B. Sands, I. J. LeGrice, B. H. Smaill, Laminar arrangement of ventricular myocytes influences electrical behavior of the heart, Circulation Research 101 (10) (2007) e103-112-e103-112.

[16] J. G. Stinstra, B. Hopenfeld, R. S. Macleod, Using models of the passive cardiac conductivity and full heart anisotropic bidomain to study the epdicardial potentials in ischaemia, in: Proceedings of the IEEE Engineering in Medicine and Biology 26th Annual International Conference, IEEE EMBS, IEEE Press, 2004, pp. 3555-3558.

[17] B. M. Johnston, S. Coveney, E. T. Y. Chang, P. R. Johnston, R. H. Clayton, Quantifying the effect of uncertainty in input parameters in a simplified bidomain model of partial thickness ischaemia, Medical and Biological Engineering and Computing (2017) DOI: $10.1007 / \mathrm{s} 11517-017-1714-\mathrm{y}$.

[18] E. T. Y. Chang, M. Strong, R. H. Clayton, Bayesian sensitivity analysis of a cardiac cell model using a Gaussian Process emulator, PLoS ONE 10 (6) (2015) e0130252.

[19] J. P. Barnes, Mathematically modeling the electrophysiological effects of ischaemia in the heart, Ph.D. thesis, Griffith University, Brisbane, Australia (2013).

[20] P. R. Johnston, A finite volume method solution for the bidomain equations and their application to modelling cardiac ischaemia, Computer Methods in Biomechanics and Biomedical Engineering 13 (2) (2010) 157-170.

[21] R. Coronel, F. J. G. Wilms-Schopman, T. Opthof, F. J. L. van Capelle, M. J. Janse, Injury current and gradients of diastolic stimulation threshold, TQ potential, and extracellular potassium concentration during acute regional ischemia in the isolated perfused pig heart, Circulation Research 68 (1991) 12411249 .

[22] M. Potse, R. Coronel, A. R. LeBlanc, A. Vinet, The role of extracellular potassium transport in computer models of the ischemic zone, Medical and Biological Engineering and Computing 45 (12) (2007) 1187-1199.

[23] P. Colli-Franzone, L. Guerri, Spreading of excitation in 3-D models of the anisotropic cardiac tissue I: Validation of the eikonal model, Mathematical Biosciences 113 (1993) 145-209.

[24] SCIRun: A Scientific Computing Problem Solving Environment, Scientific Computing and Imaging Institute (SCI). [link]. URL http://www.scirun.org

[25] W. Becker, J. E. Oakley, C. Surace, P. Gili, J. Rowson, K. Worden, Bayesian sensitvity analysis of a nonlinear finite element model, Mechanical Systems and Signal Processing 32 (2012) $18-31$.

[26] H. Abdi, Partial least squares regression (PLS-regression), Encyclopedia for research methods for the social sciences, Sage, 2003, pp. 792-795.

[27] E. Sobie, Parameter sensitivity analysis in electrophysiological models using multivariate regression, Biophysical Journal 96 (2009) 1264-1274.

[28] P. Geladi, B. R. Kowalski, Partial least squares regression: a tutorial, Analytica Chimica Acta 185 (1986) 1-17.

[29] A. G. Kléber, M. J. Janse, F. J. L. van Capelle, D. Durrer, Mechanism and time course of $\mathrm{S}-\mathrm{T}$ and $\mathrm{T}-\mathrm{Q}$ segment changes during acute regional myocardial ischemia in the pig heart determined by extracellular and intracellular recordings, Circulation Research 42 (5) (1978) 603-613. 
[30] J. Stinstra, B. Hopenfeld, R. MacLeod, On the passive cardiac conductivity, Annals of Biomedical Engineering 33 (12) (2005) 1743-1751.

[31] L. Clerc, Directional differences of impulse spread in trabecular muscle from mammalian heart, Journal of Physiology 255 (1976) 335-346.

[32] D. Swenson, J. Stinstra, B. Burton, K. Aras, L. Healy, R. MacLeod, Evaluating the effects of border zone approximations with subject specific ischemia models, in: O. Dössel, W. C. Schlegel (Eds.), IFMBE Proceedings, Vol. 25/IV, World Congress on Medical Physics and Biomedical Engineering, Springer, 2009, pp. 1680-1683. 